\title{
Noradrenergic neuromodulation of human attention for emotional and neutral stimuli
}

\author{
Benedetto De Martino • Bryan A. Strange • \\ Raymond J. Dolan
}

Received: 29 August 2007 / Accepted: 5 November 2007 / Published online: 30 November 2007

(C) Springer-Verlag 2007

\begin{abstract}
Introduction Norepinephrine (NE) has a regulatory role in human attention.

Objective To examine its role in emotional modulation of attention, we used an attentional blink (AB) paradigm, in the context of psychopharmacological manipulation, where targets were either emotional or neutral items.

Results and discussion We report behavioural evidence that $\beta$-adrenergic blockade with propranolol impairs attention independent of target valence. Furthermore, this effect is centrally mediated as administration of the peripheral $\beta$ adrenergic antagonist nadolol did not impair attention. By contrast, increasing NE tone, using the selective NE reuptake inhibitor reboxetine, improves detection of emotional stimuli.

Conclusion In line with theoretical and animal models, these findings provide human behavioural evidence that the adrenergic system has a modulatory influence on selective attention that in some instances depends on item valence.
\end{abstract}

Keyword Norepinephrine - Attentional blink paradigm . Locus coeruleus

\section{Introduction}

Norepinephrine (NE) is a monoaminergic neurotransmitter released by ascending projections of the locus coeruleus

B. De Martino $(\bowtie) \cdot$ B. A. Strange $\cdot$ R. J. Dolan

Functional Imaging Laboratory, Institute of Neurology,

Wellcome Trust Centre for Neuroimaging,

12 Queen Square,

London WC1N 3BG, UK

e-mail: b.martino@fil.ion.ucl.ac.uk
(LC). NE plays a critical role in regulating cortical function and is implicated in the pathophysiology of major psychiatric disorders, such as depression, schizophrenia, and more recently attention deficit disorder (Beane and Marrocco 2004; Pliszka et al. 1996). While the role of NE system in arousal is well established (Jouvet 1969; Robinson and Berridge 1993), recent data from animal models suggest a role in facilitating processing of relevant, or salient, information (Berridge and Waterhouse 2003; Yu and Dayan 2005). Electrophysiological studies in monkeys indicate that there are two modes by which LC activity modulates attention (Aston-Jones and Cohen 2005). Phasic LC neuronal activation is evoked by salient or goal-relevant stimuli during vigilance tasks, a response positively associated with outcomes in task-related decisions and coupled with highly accurate responses (Aston-Jones et al. 1994). Phasic responses occur against the background of, and are in turn modulated by, tonic LC activity that correlates with general arousal levels (Aston-Jones et al. 1991, 1994).

We have recently shown (Strange and Dolan 2007) that the cortical circuitry engaged by salient stimuli is inhibited by pharmacological blockade of the noradrenergic system. Specifically, we demonstrated a role for $\beta$-adrenergic receptors in this process. This differs from previous psychopharmacological experiments addressing NE modulation of attention, which have typically examined the effects of $\alpha 2$-adrenergic receptor agonists such as clonidine (Coull et al. 2001). Subjects receiving clonidine, compared to placebo, are impaired at target discrimination (Clark et al. 1986) but also report subjective withdrawal and difficulties with concentration. Clonidine acts on presynaptic $\alpha 2$-adrenergic auto-receptors to inhibit NE release; thus, clonidine-induced impaired attention may reflect decreased arousal or, due to presynaptic inhibition of NE release, decreased stimulation of predominantly postsynaptic $\alpha_{1}$ - or 
$\beta$-adrenergic receptors. Given that the $\beta_{1} \beta_{2}$-adrenergic antagonist propranolol is non-sedating (Harmer et al. 2001), its administration enables investigation of the adrenergic role in attention without sedation and localising specific attentional effects to a specific receptor subtype.

To investigate the noradrenergic modulation of human attention, we used a modification of the RSVP attentional blink (AB) paradigm in which subjects view a rapid sequence of visual stimuli (RSVP; Raymond et al. 1992) with a task requirement being to identify embedded targets. A classical finding using this type of manipulation is that identifying a first target stimulus (T1) causes transient impairment in detecting a subsequently presented second target (T2), an effect reduced with increasing temporal lag between the two targets. This paradigm allowed us to investigate how emotional stimuli modulate the allocation of limited attentional resources in time. Thus, the present study was designed to investigate the time dynamic of the interaction between attention and emotion.

Of particular interest to the study of a adrenergic role in attentional control is the recent finding that the $\mathrm{AB}$ can be modulated by the emotional significance of stimuli. The crucial observation in this study is that an arousing T2 stimulus is detected more often than a neutral T2 (Anderson and Phelps 2001), an effect abolished by amygdala damage (Anderson and Phelps 2001). These observations parallel those seen during studies of human episodic memory where memory is enhanced for emotional events, an effect not seen in patients with amygdala lesions (Cahill et al. 1994; Strange et al. 2003). Enhanced emotional memory effect are abolished by propranolol (Cahill et al. 1994; Strange et al. 2003), which leads to an hypothesis that the emotional modulation of the $\mathrm{AB}$ would also be abolished by $\beta$ adrenergic blockade. However, in light of our finding (Strange and Dolan 2007) that propranolol modulates the cortical circuitry involved in salience detection, the effects of propranolol on the $\mathrm{AB}$ might be more complex, i.e., there may be a generic effect of $\beta$-adrenergic blockade on $\mathrm{T} 2$ detection.

Thus, within the current RSVP task, each trial consisted of 15 words, 2 targets (bright green) and 13 distractors (black), presented for $130 \mathrm{~ms}$ each and immediately followed by the subsequent stimulus (Fig. 1). In half the trials, the T2 stimuli consisted of arousing words (e.g. rape and incest) and in the other half neutral words. Task difficulty was manipulated by introducing different temporal lags. Before the first experiment, subjects received either propranolol or placebo in a double-blind fashion (Experiment 1). In Experiment 2, subjects performed a more difficult version of the task, to address possible ceiling effects seen in Experiment 1. As an additional manipulation, subjects were randomised to receive either placebo, propran-

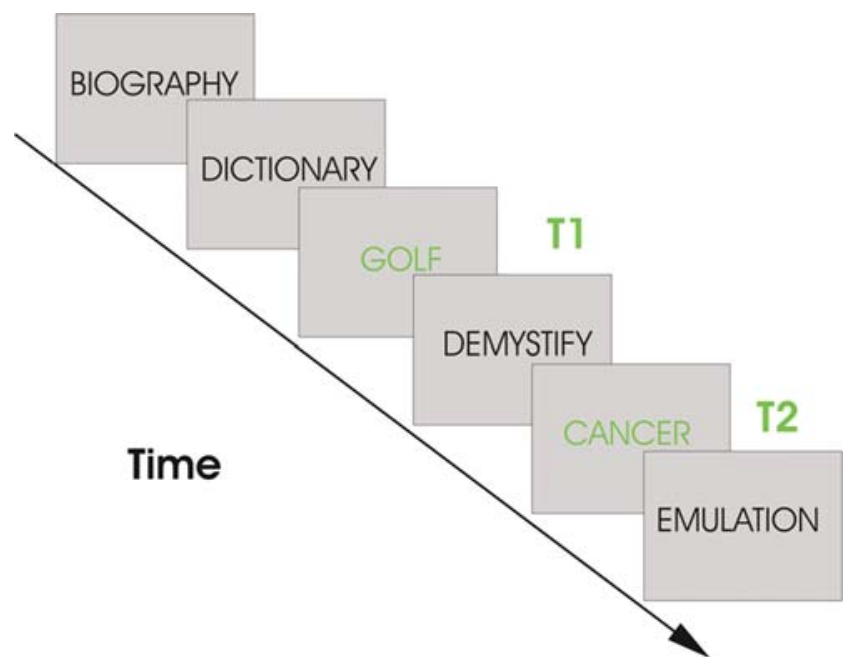

Fig. 1 Schematic diagram of the AB task. Each trial consisted of 15 words [2 targets (bright green) and 13 distractors (black)] each presented for $130 \mathrm{~ms}$ (Experiment 1) or $110 \mathrm{~ms}$ (Experiment 2) and immediately followed by the subsequent stimulus. In half the trials, the T2 stimuli consisted of an arousing word (e.g. rape and incest) and in the other half T2 were neutral words (e.g. pepper and omit). The neutral and emotional stimuli were matched for average word length, word frequency and interletter frequency. Order or presentation of emotional and neutral trials was random. The temporal lag between the first target $(T 1)$ and the second target $(T 2)$ was variable. At early lag $(<500 \mathrm{~ms}$, SOA), the detection of target T2 is more susceptible to the attentional blink compared with late lag ( $>500 \mathrm{~ms}$, SOA). The subjects' task was to monitor the RSVP stream and report both coloured green targets (T1 and $\mathrm{T} 2$ ) by typing them at the end of a stimulus sequence

olol or the selective norepinephrine reuptake inhibitor (SNRI) reboxetine. Thus, this experimental design enabled us to investigate the effects of adrenergic modulation, either inhibitory or facilitatory, on an attention paradigm in which both the salience and valence of the T2 target was manipulated. In a third experiment, we tested for the contribution of peripheral $\beta$-blockade to the attentional effects seen with propranolol by administering nadolol, a $\beta$-blocker that does not cross the blood-brain barrier (BBB). Experiment 3 also included a group that received a lower dose of propranolol to determine whether modulatory effects observed in Experiments 1 and 2 were dose dependent.

\section{Materials and methods}

Ninety-six healthy right-handed native English-speaking subjects took part in our studies. All volunteers gave informed consent, were free of neurological, psychiatric and physical illness and had not been on any medication for $>3$ months. The study was approved by the National Hospital for Neurology and Neurosurgery and the Institute of Neurology Joint Research Ethics Committee. 


\section{Experiment 1}

Subjects In a double-blind experimental design, 36 subjects [18 male, mean age $(\mathrm{SD})=24.2$ (3.9); 18 female, mean age $=$ 23.1 (4.0)] received either a 40-mg oral dose of propranolol hydrochloride (a $\beta 1$ and $\beta 2$ adrenergic receptor antagonist) or a $100 \mathrm{mg}$ oral dose of ascorbic acid (placebo pill). Drug allocation, in this and subsequent experiments, was balanced for gender. In view of the kinetics of propranolol's peak plasma concentration (1-2 h), the attentional blink task commenced $90 \mathrm{~min}$ after drug administration. Blood pressure (BP) was measured immediately before drug administration (time $0 \mathrm{~min}$ ) and the attention task (time +90 $\mathrm{min}$ ).

Stimuli We used a modification of the RSVP paradigm (Anderson and Phelps 2001; Raymond et al. 1992) where each trial consisted of 15 words [2 targets (bright green) and 13 distractors (black)] each presented for $130 \mathrm{~ms}$ and immediately followed by the subsequent stimulus (Fig. 1). Six trial lags were introduced from lag 2 [1 distractor presented between the two targets (T1-T2) SOA=260 ms] to lag 7 [6 distractors presented between the two targets (T1-T2) $\mathrm{SOA}=910 \mathrm{~ms}]$. In half the trials, the T2 stimuli consisted of an arousing word (e.g. rape and incest), and in the other half, T2 were neutral words (e.g. pepper and omit). The neutral and emotional stimuli were matched for average word length, word frequency and inter-letter frequency. The presentation order of emotional and neutral trials was random. The subjects' task was to monitor the RSVP stream and report both coloured green targets (T1 and T2) by typing them at the end of a stimulus sequence (trials in which a T1 target was not reported correctly were discarded from the analysis of T2 effects).

Statistics Following the analysis of Anderson and Phelps (Anderson and Phelps 2001), data were segregated into early (lags 2-3, 260-390 ms) and late lag (lags 6-7, 780$910 \mathrm{~ms}$ ). We performed a $2 \times 2 \times 2 \mathrm{drug}$ (propranolol $40 \mathrm{mg}$, placebo) $\times$ valence of T2 stimulus (emotional, neutral) $\times$ lag (early vs late) analysis of variance (ANOVA). In this and all subsequent experiments, ANOVAs were followed by post-hoc two sample unequal variance $t$ tests to test for simple effects.

\section{Experiment 2}

Subjects In a double-blind experimental design, 30 subjects [15 male, mean age $(\mathrm{SD})=24.7(2.8) ; 15$ female, mean age $=$ 23.3 (3.1)] were allocated to one of three equal sized groups and received either a 40-mg oral dose of propranolol, a 4-mg oral dose of reboxetine methansulphonate (a selective norepinephrine reuptake inhibitor) or a 100-mg oral dose of ascorbic acid (placebo pill). In view of the kinetics of propranolol and reboxetine's peak plasma concentration (12 and $1.5 \mathrm{~h}$, respectively), the attentional blink task commenced $120 \mathrm{~min}$ after drug administration. One subject in the propanolol group was excluded from further analysis because behavioural performance was more than two standard deviations below the group average.

Stimuli The task used was identical to Experiment 1 except for two critical modifications; the time of the stimulus presentation was decreased from $130 \mathrm{~ms}$ in Experiment 1 to $110 \mathrm{~ms}$, and targets were separated by six different time lags ranging from one to nine distractors presented between the two targets (lags 2-3-4-7-8-9) [e.g. lag 2 (T1-T2) $\mathrm{SOA}=220 \mathrm{~ms}$; lag $9(\mathrm{~T} 1-\mathrm{T} 2) \mathrm{SOA}=990 \mathrm{~ms}]$.

Statistics Data were collapsed into early (lags 2-3-4, 220$440 \mathrm{~ms}$ ) and late lags (lags 7-8-9, 770-990 ms) and a drug (propranolol $40 \mathrm{mg}$, reboxetine, placebo) $\times \mathrm{T} 2$ valence (emotional, neutral) $\times$ lag (early, late) $3 \times 2 \times 2$ ANOVA performed. For each drug group, we also performed a $2 \times 2$ drug (drug, placebo) $\times$ T2 valence (emotional, neutral) ANOVA.

\section{Experiment 3}

Subjects In a double-blind experimental design, 30 subjects [15 male, mean age $(\mathrm{SD})=25.2$ (3.7); 15 female, mean age $=$ 25.5 (3.9)] were allocated to one of three equal size groups and received either a $20-\mathrm{mg}$ oral dose of propranolol, a 40mg oral dose of nadolol (a $\beta 1$ and $\beta 2$ adrenergic receptor antagonist that does not cross the $\mathrm{BBB}$ ) or a $100 \mathrm{mg}$ oral dose of ascorbic acid (placebo pill). In view of the kinetics of propranolol and nadolol peak plasma concentration (1-2 h), the attentional blink task commenced $120 \mathrm{~min}$ after drug administration. One subject in the propanolol group was excluded from further analysis because performance was more than two standard deviations below the group average (task outlier). Three subjects in the placebo group were excluded (two on the grounds of being task outliers and one on subsequent disclosure of not being a native English speaker).

Stimuli The task used was identical to Experiment 2 using the same equipment.

Statistics After exclusion criteria, the size of the placebo group was drastically reduced (seven subjects). Thus, to retain sufficient statistical power, we collapsed data from 
the current placebo group with the placebo group from Experiment 2. Data were collapsed into early (lags 2-3-4, 220-440 ms) and late lags (lags 7-8-9, 770-990 ms) and separate analyses performed for each drug group (propanolol $20 \mathrm{mg}$, nadolol $40 \mathrm{mg}$ ) vs placebo, i.e. separate group $($ drug $\times$ placebo $) \times \mathrm{T} 2$ valence (emotional, neutral $) \times$ lag (early, late) $2 \times 2 \times 2$ ANOVAs.

\section{Results}

Experiment 1 This psychopharmacological experiment demonstrated that $\beta$-adrenergic blockade by propranolol significantly impaired detection of $\mathrm{T} 2$ targets independently of their emotional valence (i.e. emotional and neutral; see Fig. 2). In both placebo and drug groups, we observed enhanced reporting of emotional, relative to neutral, T2 stimuli and a proportional increase in detecting both types of T2 stimuli with increasing T1-T2 lag, in agreement with previous observations (Anderson and Phelps 2001). In the placebo group, the percentage of T2 correctly reported at early lag was for the neutral T2 (NT2) $86.99 \% \pm 2.42$ and for the emotional T2 (ET2) $93.93 \% \pm 1.43$. At late lag, these values increased to NT2 $=92.62 \% \pm 1.28$ and ET2 $=95.8 \% \pm$
1.13. Conversely, in the 40-mg propranolol group, the percentage of T2 correctly reported at early lag was NT2 $=$ $80.69 \% \pm 1.90$ and ET2 $=91.26 \% \pm 1.93$; performance at late lag increased to NT2 $=88.18 \% \pm 1.90$ and ET2 $=93.84 \% \pm$ 1.47 .

A drug (propranolol, placebo) $\times \mathrm{T} 2$ valence (neutral, emotional) $\times$ lag (early, late) $2 \times 2 \times 2$ ANOVA yielded significant main effects of treatment $\left[F_{(1,34)}=4.98, P<\right.$ $0.05]$, T2-valence $\left[F_{(1,34)}=51.31, P<0.0001\right]$ and lag $\left[F_{(1,34)}=26.33, P<0.0001\right]$. In line with the observation of Anderson and Phelps that affective modulation of the attentional blink is most pronounced at early lags, we demonstrated a lag $\times$ T2-valence interaction significant at trend level $\left[F_{(1,34)}=3.72 P=0.062\right)$. Critically, there was no significant interaction between T2-valence and drug manipulation $\left[F_{(1,34)}=2.65, P=0.29\right]$ indicating that a propranolol effect was not influenced by T2 target valence (Fig. 2). The simple effect of group (placebo, propranolol) on T2 reporting was significant for neutral T2 $\left[t_{(1,19)}=2.04, P<\right.$ 0.05 one-tailed $]$ but not for emotional T2 $\left[t_{(1,19)}=1.13, P=\right.$ $0.13]$.

Furthermore, no significant interaction was found between lags (early, late) and drug manipulation (propranolol, placebo) $\left[F_{(1,34)}=0.51, P=0.476\right)$ indicating that the effect of propranolol is present across all lags. Figure $2 \mathrm{c}$ shows
Fig. 2 Behavioural results of Experiment 1. Subpanels a and b show the percentage (\%) of T2 stimuli that are correctly reported for each T1-T2 temporal lag for $\mathbf{a}$ placebo and $\mathbf{b}$ propranolol $(40 \mathrm{mg})$ groups. In both groups, T2 detection improves with increasing temporal lags, and is significantly higher for emotional T2 (red squares) compared with neutral T2 (blue diamonds). The administration of propranolol significantly impairs T2 detection independently of the arousal content of the stimuli. c Impaired emotional (red lines) and neutral (blue lines) T2 detection produced by propranolol (dashed lines) relative to placebo (solid lines) is more marked at early lags (260-390 ms, SOA), when attentional demand is higher, compared with late lags (780-910 ms, SOA). In particular, the effect of the propanolol on the detection of neutral target was most dramatic at lag 2 where the $\mathrm{AB}$ is known to be maximal
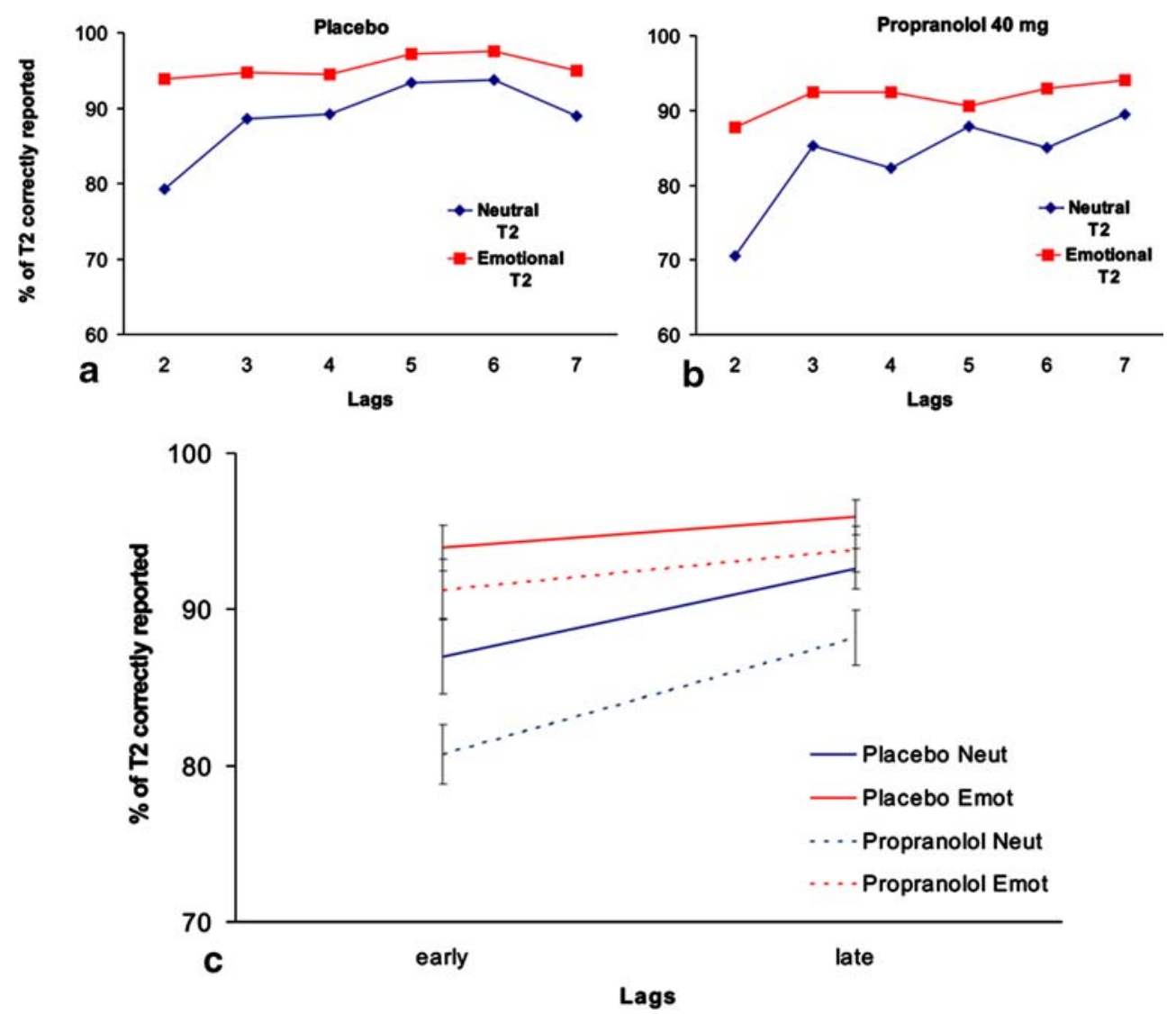
the percentage $(\% \pm \mathrm{SE})$ of correctly reported emotional and neutral T2 for early (260-390 ms, SOA) and late lags (780$910 \mathrm{~ms}, \mathrm{SOA})$. Importantly, performance on $\mathrm{T} 1$ target detection was not statistically different between placebo $(96.27 \%)$ and propranolol $(96.18 \%)$ groups $\left[\mathrm{t}_{(1,19)}=0.17\right.$, $P>0.05$ one-tailed independent $t$ test].

As seen in Fig. 2, performance in both placebo and drug groups at late lags approached ceiling levels, which may have obscured differential effects at these later lags. Thus, in Experiment 2, we reduced the interstimulus interval, making T2 detection more difficult. Removing this ceiling effect enabled us to test the additional hypothesis that increasing NE levels, with the SNRI reboxetine, would enhance $\mathrm{T} 2$ detection.

Finally, the mean BP at the time of placebo/drug (0 min) administration for the placebo group was $90 \mathrm{mmHg}$ and at the start of the experiment (+90 min) was $88.5 \mathrm{mmHg}$ $\left[t_{(1,17)}=0.95 \mathrm{P}=0.35\right.$ two-tailed paired $t$ test $]$. For the propranol group at $0 \mathrm{~min}$ was $86.2 \mathrm{mmHg}$ and showed a significant reduction at (+90 $\mathrm{min}) 81.9 \mathrm{mmHg}\left[t_{(1,17)}=2.16\right.$ $\mathrm{P}<0.05$ two-tailed paired $t$ test].

Experiment 2 Three different drug groups (placebo, propranolol and reboxetine) performed an attentional blink task identical to Experiment 1 except for a reduction in stimulus presentation time from 130 to $110 \mathrm{~ms}$ and a subtle change in lag range. This experiment confirms the previous finding (from Experiment 1) of a propranolol-induced impaired detection of $\mathrm{T} 2$ targets, independently of whether these are emotional or neutral stimuli. It also demonstrates that reboxetine selectively boosts the detection of emotional T2 with no effect on the neutral T2 (Fig. 3). In the placebo group, the percentage of $\mathrm{T} 2$ correctly reported at early lag was NT2 $=64.02 \% \pm 4.67$ and ET2 $=74.26 \% \pm 5.65$, and at late lag NT2 $=77.87 \% \pm 4.56$ and ET2 $=84.5 \% \pm 4.99$. In the $40 \mathrm{mg}$ propranolol group, the percentage of T2 correctly reported at early lag was NT2 $=46.1 \% \pm 5.28$ and ET2 $=$ $61.6 \% \pm 5.93$ conversely at late lag NT2 $=62.68 \% \pm 7.69$ and $\mathrm{ET} 2=70.48 \% \pm 7.62$. In the reboxetine group, the percentage of T2 correctly reported at early lag was NT2 $=62.8 \% \pm$ 3.65 and $\mathrm{ET} 2=82.64 \% \pm 4.18$ at late lag NT2 $=80.95 \% \pm$ 2.05 and $\mathrm{ET} 2=90.23 \% \pm 1.92$.

Figure $3 \mathrm{c}$ shows the percentage $(\% \pm \mathrm{SE})$ of correctly reported emotional (E) and neutral $(\mathrm{N}) \mathrm{T} 2$ for early (220-440 ms, SOA) and late lags (770-990 ms, SOA). A drug (placebo, propranolol, reboxetine) $\times \mathrm{T} 2$ valence (neutral, emotional) $\times$ lag (early, late) $3 \times 2 \times 2$ ANOVA yielded a significant main effect of drug $\left[F_{(1,27)}=5.44\right.$, $P<0.01]$, T2-valence $\left[F_{(1,27)}=73.8, P<0.0001\right]$ and lag $\left[F_{(1,27)}=93.54, P<0.0001\right]$. The three-way interaction of drug $\times$ T2-valence $\times$ lag was also significant $\left[\mathrm{F}_{(2,27)}=3.46\right.$, $P<0.05]$.
To investigate the effects of drug relative to placebo, we performed two further $2 \times 2 \times 2$ ANOVAs. The effect of propranolol in a drug (placebo, propranolol) $\times \mathrm{T} 2$-valence (neutral, emotional) $\times$ lag (early, late) $2 \times 2 \times 2$ ANOVA yielded a significant main effect of drug manipulation $\left[F_{(1,18)}=4.58, P<0.05\right]$, T2-valence $\left[F_{(1,18)}=24.87, P<\right.$ $0.0001]$ and lag $[F(1,18)=86.51, P<0.0001]$. The interaction between T2-valence and drug manipulation was not significant $\left[F_{(1,18)}=0.62, P=0.44\right]$. The simple effects of group (placebo, propranolol) on $\mathrm{T} 2$ detection was significant for neutral T2 $\left[t_{(1,19)}=2.53, P<0.01\right)$ and at trend level for emotional T2 $\left[t_{(1,19)}=1.53, P=0.07\right]$. These data replicate the results of Experiment 1, confirming that propranolol impairs T2 detection independently of T2 emotional valence. As in Experiment 1, the degree of affective modulation was most pronounced at early lags, evident in a significant interaction of lag $\times$ T2-valence $\left[F_{(1,18)}=6.59, P<0.05\right]$. Again, no significant interaction was found between lags (early, late) and drug manipulation (propranolol, placebo) $\left[F_{(1,18)}=0.05, P=0.862\right]$. Finally, in this more difficult version of our task, T1 detection is impaired in the propranolol group relative to placebo [82.13 vs $89.28 \%$ correct, respectively; $t_{(1,18)}=1.84, P<0.05$ onetailed independent-samples $t$ test].

By contrast, reboxetine evoked a selective enhancement in emotional T2 detection. A drug (placebo, reboxetine) $\times$ $\mathrm{T} 2$ valence (neutral, emotional) $\times$ lag (early, late) $2 \times 2 \times 2$ ANOVA yielded a significant main effect of T2 valence $\left[\mathrm{F}_{(1,18)}=107.89, P<0.0001\right]$ and $\operatorname{lag}\left[\mathrm{F}_{(1,18)}=54.39, P<\right.$ $0.0001]$, but the main effect of reboxetine relative to placebo was not significant $\left[F_{(1,18)}=0.57, P=0.46\right]$. However, the interaction between T2-valence and drug manipulation was significant $\left[F_{(1,18)}=10.61, P<0.005\right)$. The simple effect of group (placebo, reboxetine) on $\mathrm{T} 2$ detection was not significant for neutral $\mathrm{T} 2\left[t_{(1,19)}=0.44\right.$, $P=0.66]$ and was at trend level for emotional T2 $\left[t_{(1,19)}=\right.$ $1.54, P=0.07]$. These data demonstrate that enhanced NE levels selectively improves detection of an emotional T2 stimulus with no effect on neutral T2. Again, detection of emotional relative to neutral $\mathrm{T} 2$ stimuli was greatest at early lags for both groups indexed by a significant lag $\times$ T2valence $\left[F_{(1,18)}=22.8, P<0.001\right]$ interaction. By contrast, a lag (early, late) $\times$ drug manipulation (reboxetine, placebo) interaction was not significant $\left[F_{(1,18)}=0.036, P=0.852\right]$, indicating that the behavioural effect of reboxetine is present across all lags. $\left[t_{(1,17)}=0.95, P=0.35\right.$ two-tailed paired $t$ test).

The mean BP at the time of placebo/drug $(0 \mathrm{~min})$ administration for the placebo group was $90.9 \mathrm{mmHg}$ and showed a significant reduction at $(+90 \mathrm{~min}) 82.9 \mathrm{mmHg}$ $\left[t_{(1,9)}=2.79, P<0.05\right.$ two-tailed paired $t$ test $]$. For the reboxetine group at $(0 \mathrm{~min})$ was $91.3 \mathrm{mmHg}$ and showed a non-significant reduction at $(+90 \mathrm{~min}) 90.4 \mathrm{mmHg}$ 
Placebo
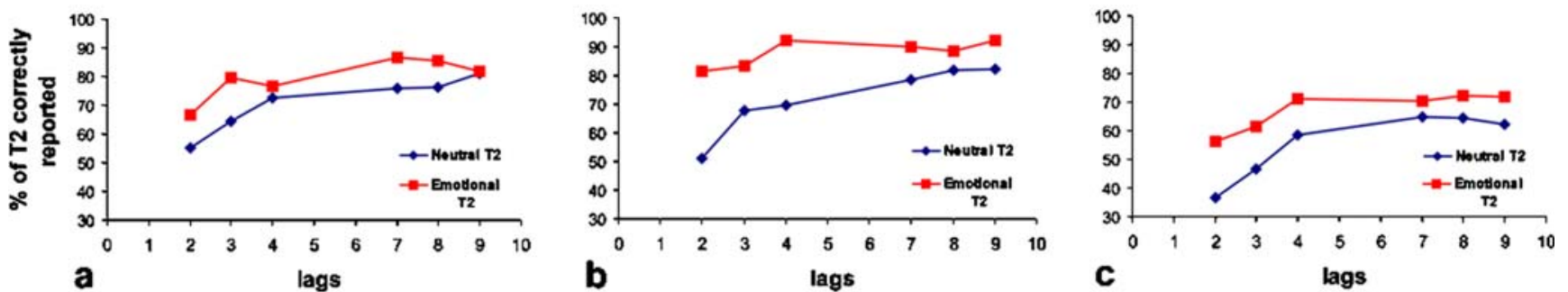

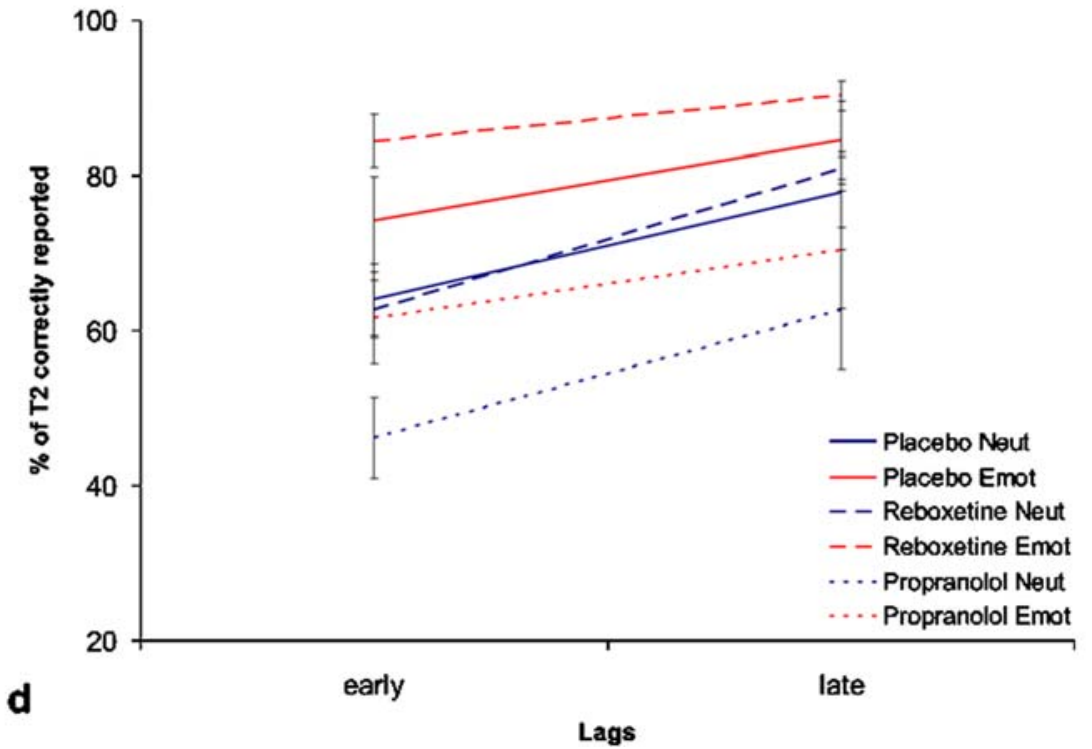

Fig. 3 Behavioural results of Experiment 2. Subpanels a-c show the percentage of $\mathrm{T} 2$ stimuli that are correctly reported for each $\mathrm{T} 1-\mathrm{T} 2$ temporal lag respectively in the placebo, reboxetine and propranolol groups. In all groups, 72 detection improved with increasing temporal lags and is significantly enhanced for emotional (red squares) relative to neutral (blue diamonds) T2 detection. b As in Experiment 1, propranolol significantly impairs $\mathrm{T} 2$ detection independently of

$\left[t_{(1,9)}=0.69, P=0.50\right.$ two-tailed paired $t$ test $]$. Finally, in the propranolol group, the mean BP at (0 min) was 85.1 and showed a significant reduction at $(+90 \mathrm{~min}) 78.9 \mathrm{mmHg}$ $\left[t_{(1,9)}=3.97, P<0.005\right.$ two-tailed paired $t$ test $]$.

Experiment 3 To further investigate the effects of propranolol, we conducted a third experiment in which three different drug groups (placebo, nadolol $40 \mathrm{mg}$ and propranolol $20 \mathrm{mg}$ ) performed the $\mathrm{AB}$ task identical to Experiment 2. The first drug group received $40 \mathrm{mg}$ of nadolol, a beta blocker with a the same mechanism of action of propranolol (i.e. selective blockers of $\beta 1$ and $\beta 2$ adrenergic receptors) but that critically does not pass the BBB (Hardman et al. 2001). This manipulation controlled for the potential contribution of peripheral $\beta$-blockade to the attentional effects observed with propranolol. The second group received a dose of propranolol $(20 \mathrm{mg})$ that arousal. c Conversely, reboxetine significantly enhances detection of emotional T2 with no effect on neutral T2. d T2 target detection in placebo (solid lines), propranolol (small dashed lines) and reboxetine (large dashed lines) groups on early lag (220-440 ms, SOA), when the attentional demand is higher, compared with late lags (770 $990 \mathrm{~ms}, \mathrm{SOA}$ ) for emotional (red lines) and neutral (blue lines) T2 stimuli

was half that used in Experiments 1 and 2. This treatment was aimed at determining whether a lower dose of propranolol would result in an attentional effect that was specific for target valence. As shown in Fig. 4, correct T2 detection at early lag in the placebo group was NT2= $59.19 \% \pm 3.44$ and ET2 $=75.32 \% \pm 3.58$, and at late lag NT2 $=$ $76.45 \% \pm 3.13$ and ET2 $=86.68 \% \pm 3.21$. In the $20 \mathrm{mg}$ propranolol group the percentage of $\mathrm{T} 2$ correctly reported at early lag was NT2 $=65.40 \% \pm 6.40$ and ET2 $=78.20 \% \pm 6.31$, whereas at late lag NT2 $=80.43 \% \pm 5.02$ and ET2 $=87.62 \% \pm$ 4.27. In the $40 \mathrm{mg}$ nadolol group, the percentage of $\mathrm{T} 2$ correctly reported at early lag was NT2 $=62.24 \% \pm 4.76$ and ET2 $=76.82 \% \pm 5.57$, and at late lag NT2 $=76.59 \% \pm 4.08$ and $\mathrm{ET} 2=88.07 \% \pm 3.69$.

Thus, T2 detection did not differ between nadolol and placebo groups (Fig. 4). The drug (placebo, nadolol) $\times \mathrm{T} 2$ 

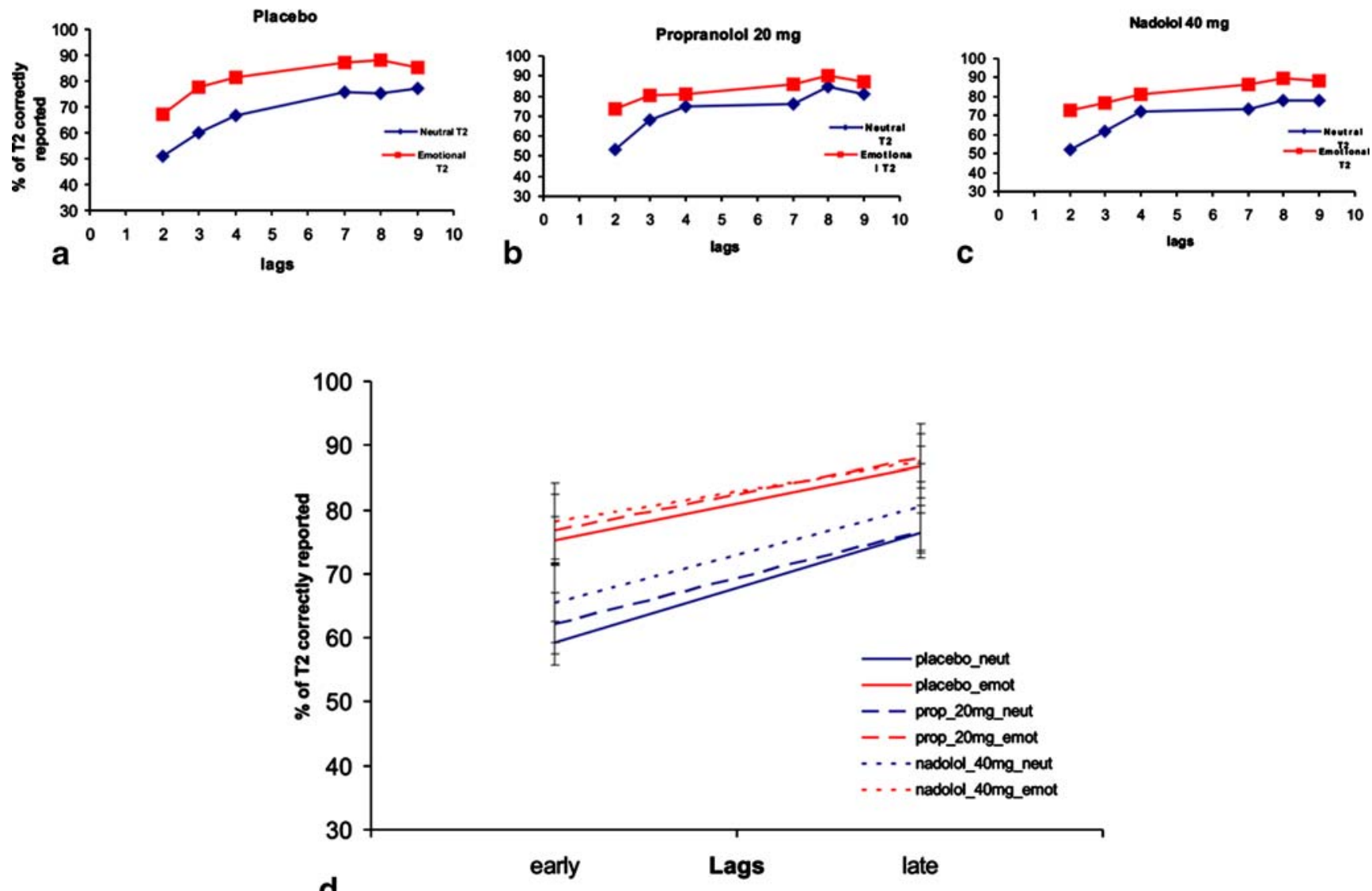

Fig. 4 Behavioural results of Experimental 3. Subpanels a-c show the percentage of $\mathrm{T} 2$ stimuli that are correctly reported for each T1-T2 temporal lag respectively in the placebo, nadolol and propranolol low dose $(20 \mathrm{mg})$ groups. In all groups, T2 detection improved with increasing temporal lags and is significantly enhanced for emotional (red squares) relative to neutral (blue diamonds) T2 detection. Both

propanolol $20 \mathrm{mg}$ (b) and nadolol $40 \mathrm{mg}$ (c) do not show significant differences from the placebo group. d T2 target detection in placebo (solid lines), nadolol $40 \mathrm{mg}$ (small dashed lines) and propanolol $20 \mathrm{mg}$ (large dashed lines) groups on early lag (220-440 ms, SOA), when the attentional demand is higher, compared with late lags (770-990 ms, SOA) for emotional (red lines) and neutral (blue lines) T2 stimuli

valence (neutral, emotional) $\times$ lag (early, late) $2 \times 2 \times 2$ ANOVA yielded a significant main effect of $\mathrm{T} 2$ valence $\left[F_{(1,25)}=89.16, P<0.0001\right]$ and lag $\left[F_{(1,25)}=12.45, P<\right.$ $0.005]$ ), but no significant main effect of drug relative to placebo $\left[F_{(1,25)}=0.06, P=0.80\right]$ or drug $\times$ T2-valence interaction $\left[F_{(1,25)}=1.48, P=0.23\right]$ (Fig. 4). These results confirm that the modulatory effects of propranolol observed in Experiments 1 and 2 are due to central, and not peripheral, effects.

Performance in the low dose propanolol group $(20 \mathrm{mg})$ was equivalent to that of the placebo group. The drug (placebo, propanolol low dose $20 \mathrm{mg}$ ) $\times \mathrm{T} 2$ valence (neutral, emotional) $\times$ lag (early, late) $2 \times 2 \times 2$ ANOVA yielded a significant main effect of $\mathrm{T} 2$ valence $\left[F_{(1,24)}=\right.$ 49.33, $P<0.0001]$ and lag $\left[F_{(1,24)}=68.99, P<0.0001\right]$. Although the low dose propranolol group $(20 \mathrm{mg})$ performed the task slightly better than the placebo group, this difference was not statistically significant [main effect of drug relative to placebo $\left.F_{(1,24)}=0.42, P=0.52\right]$. Critically, there was no significant interaction between drug and T2arousal $\left[\mathrm{F}_{(1,24)}=1.08, \mathrm{P}=0.31\right]$ (Fig. 4). Thus, only the

higher propranolol dose (40 mg) impairs target detection, an effect that is independent of target arousal content. A lower propranolol dose does not impair detection of neutral or emotional $\mathrm{T} 2$ detection.

The mean BP at the time of placebo/drug $(0 \mathrm{~min})$ administration was for the placebo group was $90.5 \mathrm{mmHg}$ and showed a non-significant reduction at (+90 $\mathrm{min})$ $86.0 \mathrm{mmHg}\left[t_{(1,9)}=1.8, P=0.102\right.$ two-tailed paired $t$ test $]$. For the nadolol group at ( $0 \mathrm{~min}$ ) was $90 \mathrm{mmHg}$ and showed a non-significant reduction at $(+90 \mathrm{~min}) 88.6 \mathrm{mmHg}\left[t_{(1,9)}=\right.$ 0.4, $P=0.69$ two-tailed paired $t$ test]. Finally, in the propranolol group, the mean BP at $(0 \mathrm{~min})$ was 92.3 and showed a significant reduction at $(+90 \mathrm{~min}) 85.8 \mathrm{mmHg}$ $\left[t_{(1,9)}=2.79, P<0.05\right.$ two-tailed paired $t$ test $]$.

\section{Discussion}

The critical new observation we report is that $\beta$-adrenergic blockade by propranolol $(40 \mathrm{mg})$ impairs detection of $\mathrm{T} 2$ stimuli regardless of the emotional arousal of these stimuli 
(Experiment 1). This is the first demonstration that a manipulation of the adrenergic system affects the AB. We note in this regard that a recent study found no effect of clonidine on neutral T2 detection (Nieuwenhuis et al. 2007). The robustness of our reported $\beta$-adrenergic effect is evident in a replication within Experiment 2. Furthermore, these data also replicate previous observations regarding emotional modulation of the attentional blink (Anderson and Phelps 2001). Specifically, placebo and drug groups, in Experiments 1 and 2, showed enhanced T2 detection with increasing T1-T2 lag for both emotional and neutral T2, while emotional T2 stimuli were more frequently detected than neutral $\mathrm{T} 2$, an effect more pronounced at early lags.

A recent comprehensive account of the role of $\mathrm{NE}$ in attention is derived from monkey electrophysiological experiments, which indicate two modes of LC activity (Aston-Jones and Cohen 2005). Phasic LC neuronal activation is evoked by salient or goal-relevant stimuli during vigilance tasks (Aston-Jones et al. 1994). Phasic responses are evoked against the background of, and in turn modulated by, tonic LC activity that correlates with general arousal levels (Aston-Jones et al. 1991; AstonJones et al. 1994). Whereas the LC phasic response itself is relatively brief in duration (typically of 50-100 ms), the ensuing neuromodulatory effects of $\mathrm{NE}$ on target cortical areas are known to be sustained relative to the LC phasic response.

One recent hypothesis regarding the neurobiological underpinnings of the attentional blink suggests that it is a product of the temporal dynamics of the LC-NE system (Nieuwenhuis et al. 2005). Although NE may potentiate processing in cortical areas, local NE release within LC is thought to be auto-inhibitory, due to effects of $\mathrm{NE}$ at presynaptic and dendritic $\alpha_{2}$ autoreceptors (Egan et al. 1983; Washburn and Moises 1989; Williams et al. 1985). This autoinhibition results in a refractory-like period after a LC phasic response, during which a subsequent LC phasic discharge is rarely observed (Aston-Jones et al. 1994; Usher et al. 1999). This refractoriness peaks at approximately 50$100 \mathrm{~ms}$ after the LC phasic response, typically 200-250 ms after the eliciting stimulus, and usually lasts $200 \mathrm{~ms}$ or until about 400-450 ms post-stimulus. The length of the refractory period coincides with the $\mathrm{T} 1$ and $\mathrm{T} 2$ temporal lag in which the blink for the second stimulus T2 is most marked (Nieuwenhuis et al. 2005). Thus, this formulation predicts an effect of NE modulation on both emotional and neutral T2 detection.

Our behavioural effects can be explained via an extension of the aforementioned AB-NE model (Nieuwenhuis et al. 2005) in which we also manipulated the salience level of the T2 target (arousing or neutral). Previous studies demonstrate that arousing stimuli presented in different modalities induce a robust phasic discharge of NE neurons in LC (Aston-Jones and Bloom 1981; Foote and Morrison 1987). Thus, the enhanced detection of emotional relative to neutral T2 stimuli, demonstrated by Anderson and Phelps (Anderson and Phelps 2001) and replicated here in Experiments 1 and 2, can be attributable to an increase in phasic NE release elicited by emotional, relative to neutral, stimuli. Propranolol has been demonstrated to reduce the phasic response elicited by novel stimuli in rats (Kitchigina et al. 1997). The effects we report after propranolol administration may consequently be explained in terms of a reduced impact of LC phasic response to targets. A critical observation in our data is that propranolol-treated subjects show impaired T2 detection, relative to placebo, for both neutral and emotional T2. Importantly, emotional T2 items, eliciting an increase in NE release, are still detected more frequently than neutral T2 even in the presence of propranolol. According to the model we propose, the behavioral effect in target detection is relative to the magnitude of NE release, assumed to be larger for an arousing T2. Note that our task required subjects to report both T1 and T2 stimuli correctly. The NE-AB hypothesis (Nieuwenhuis et al. 2005) states that initial $\mathrm{T} 1$ detection is mediated by phasic LC activity, with the subsequent LC refractory period leading to impaired T2 detection. If propranolol inhibits phasic LC activity, it follows that $\mathrm{T} 1$ detection should also be impaired by betablockade. In Experiment 2, in which an increase in task difficulty prevented $\mathrm{T} 1$ detection ceiling effects, we indeed showed a significant impairment for $\mathrm{T} 1$ detection for the propranolol compared with the placebo group. Previous evidence (Jolicoeur 1999) provides an interpretation that the increased difficulty in T1 detection under propranolol may also contribute to the increased $A B$ magnitude that is independent of T2 emotionality, as demonstrated in this study.

One of the aims of Experiment 3 was to determine whether a lower dose $(20 \mathrm{mg})$ of propranolol would yield arousal-specific effects on T2 detection. In other words, we tested whether less $\beta$-adrenergic blockade could be sufficient to abolish enhanced detection of emotional T2 targets, without affecting neutral T2 detection. We did not, however, observe any difference in $\mathrm{T} 2$ detection for either neutral or emotional targets between the propranolol $20 \mathrm{mg}$ group and placebo. Experiment 3 also served to demonstrate that administration of nadolol, a peripherally acting $\beta$-adrenergic antagonist, has no effect on T2 detection, thus indicating that the attentional effects of propanolol are centrally mediated.

In addition to demonstrating attentional impairment evoked by blocking the $\beta$-adrenergic system, administration of a SNRI in Experiment 2 also enabled examination of the attentional effects of increased NE concentration at the 
synaptic level. We provide evidence for reboxetine-evoked enhanced attention, which, in contradistinction to the impairment observed with propranolol, is dependent on the emotional salience of the target. That reboxetine failed to improve neutral $\mathrm{T} 2$ detection accords with previous data showing no effect of reboxetine on a neutral continuous performance attention task (Plewnia et al. 2006).

Reboxetine-induced enhanced emotional T2 detection can potentially be explained in terms of the NE-AB hypothesis (Nieuwenhuis et al. 2005). Previous studies demonstrate increased concentration of cortical NE, particularly in the frontal cortex, after acute reboxetine administration (Sacchetti et al. 1999). By contrast, in the LC, NE reuptake inhibitors attenuate the firing activity of LC-NE neurons via $\alpha_{2}$-adrenergic autoreceptor overactivation (Beique et al. 2000; Kasamo et al. 1996; Lacroix et al. 1991). Thus, SNRIs mediate two opposite effects: at a cortical level, they potentiate attentional gain; but at the level of LC, SNRIs increase the refractory period via presynaptic $\alpha_{2}$ receptors. These two NE influences could potentially produce opponent effects on $\mathrm{T} 2$ detection involving facilitation and impairment in the $\mathrm{AB}$ task. In Experiment 2, we found that reboxetine selectively boosted detection of emotional T2 stimuli with no effect on neutral T2. These findings can be reconciled with the proposed model if, for neutral T2 detection, the facilitatory and impairing effects of reboxetine are in relative equilibrium, resulting in no net effect on neutral target detection. Conversely, the boost in NE elicited by the arousing T2 positively interacts with this state of equilibrium thereby significantly improving target detection for emotional items.

Enhanced processing of emotional stimuli might also, in theory, result in a lowered perceptual threshold for the arousing stimuli that arises epigenetically (Weinberger 1999). This framework predicts that propranolol would not have a selective effect on the perception of emotional stimuli, as the lower threshold for processing arousing stimuli is 'hard-wired' and therefore not dependent on phasic release of noradrenaline. This model could explain the findings from Experiments 1 and 2 showing that propranolol does not have a selective effect on emotional $\mathrm{T} 2$ processing.

Our results show a dissociation in the effects of propranolol on attention from effects previously reported on episodic memory (Strange et al. 2003). Whereas the effect of propranolol is selective for the emotional modulation of memory, we demonstrate for the first time in humans (Experiments 1 and 2) that engagement of $\beta$ adrenergic receptors in the central nervous system is essential for optimal target detection independent of stimulus arousal content. Moreover, we demonstrate that the increase in NE levels (Experiment 2) selectively improves detection of arousing stimuli. These attentional effects of pharmacological manipulation of the NE system in human subjects support and extend current theoretical models of the role of NE in attention.

Acknowledgements This work was supported by a Wellcome Trust Programme Grant to R.J.D and Wellcome Trust studentship to BDM). We thank D. Kumaran and P. Dayan for helpful discussions during the analysis of the study. We thank B. Holt for the technical help. We declare that we have no competing financial interest.

\section{References}

Anderson AK, Phelps EA (2001) Lesions of the human amygdala impair enhanced perception of emotionally salient events. Nature 411:305-309

Aston-Jones G, Bloom FE (1981) Nonrepinephrine-containing locus coeruleus neurons in behaving rats exhibit pronounced responses to non-noxious environmental stimuli. J Neurosci 1:887-900

Aston-Jones G, Cohen JD (2005) An integrative theory of locus coeruleus-norepinephrine function: adaptive gain and optimal performance. Annu Rev Neurosci 28:403-450

Aston-Jones G, Chiang C, Alexinsky T (1991) Discharge of noradrenergic locus coeruleus neurons in behaving rats and monkeys suggests a role in vigilance. Prog Brain Res 88: $501-520$

Aston-Jones G, Rajkowski J, Kubiak P, Alexinsky T (1994) Locus coeruleus neurons in monkey are selectively activated by attended cues in a vigilance task. J Neurosci 14:4467-4480

Beane M, Marrocco RT (2004) Norepinephrine and acetylcholine mediation of the components of reflexive attention: implications for attention deficit disorders. Prog Neurobiol 74:167-181

Beique J, de Montigny C, Blier P, Debonnel G (2000) Effects of sustained administration of the serotonin and norepinephrine reuptake inhibitor venlafaxine: II. In vitro studies in the rat. Neuropharmacology 39:1813-1822

Berridge CW, Waterhouse BD (2003) The locus coeruleus-noradrenergic system: modulation of behavioral state and state-dependent cognitive processes. Brain Res Brain Res Rev 42:33-84

Cahill L, Prins B, Weber M, McGaugh JL (1994) Beta-adrenergic activation and memory for emotional events. Nature 371:702-704

Clark CR, Geffen GM, Geffen LB (1986) Role of monoamine pathways in attention and effort: effects of clonidine and methylphenidate in normal adult humans. Psychopharmacology (Berl) 90:35-39

Coull JT, Nobre AC, Frith CD (2001) The noradrenergic alpha2 agonist clonidine modulates behavioural and neuroanatomical correlates of human attentional orienting and alerting. Cereb Cortex 11:73-84

Egan TM, Henderson G, North RA, Williams JT (1983) Noradrenalinemediated synaptic inhibition in rat locus coeruleus neurones. J Physiol 345:477-488

Foote SL, Morrison JH (1987) Development of the noradrenergic, serotonergic, and dopaminergic innervation of neocortex. Curr Top Dev Biol 21:391-423

Hardman JG, Limbrid LE, Gilman AG (2001) Goodman \& Gilman's the pharmacological basis of therapeutics. McGraw-Hill Professional, New York

Harmer CJ, Perrett DI, Cowen PJ, Goodwin GM (2001) Administration of the beta-adrenoceptor blocker propranolol impairs the processing of facial expressions of sadness. Psychopharmacology (Berl) 154:383-389 
Jolicoeur P (1999) Concurrent response-selection demands modulate the attentional blink. J Exp Psychol Hum Percept Perform 25:1097

Jouvet M (1969) Biogenic amines and the states of sleep. Science $163: 32-41$

Kasamo K, Blier P, De Montigny C (1996) Blockade of the serotonin and norepinephrine uptake processes by duloxetine: in vitro and in vivo studies in the rat brain. J Pharmacol Exp Ther 277: $278-286$

Kitchigina V, Vankov A, Harley C, Sara SJ (1997) Novelty-elicited, noradrenaline-dependent enhancement of excitability in the dentate gyrus. Eur J Neurosci 9:41-47

Lacroix D, Blier P, Curet O, de Montigny C (1991) Effects of longterm desipramine administration on noradrenergic neurotransmission: electrophysiological studies in the rat brain. J Pharmacol Exp Ther 257:1081-1090

Nieuwenhuis S, Gilzenrat MS, Holmes BD, Cohen JD (2005) The role of the locus coeruleus in mediating the attentional blink: a neurocomputational theory. J Exp Psychol Gen 134:291-307

Nieuwenhuis S, van Nieuwpoort IC, Veltman DJ, Drent ML (2007) Effects of the noradrenergic agonist clonidine on temporal and spatial attention.Psychopharmacology (Berl).193 (2):261-269

Plewnia C, Hoppe J, Gerloff C (2006) No effects of enhanced central norepinephrine on finger-sequence learning and attention. Psychopharmacology (Berl) 187:260-265

Pliszka SR, McCracken JT, Maas JW (1996) Catecholamines in attention-deficit hyperactivity disorder: current perspectives. J Am Acad Child Adolesc Psychiatry 35:264-272
Raymond JE, Shapiro KL, Arnell KM (1992) Temporary suppression of visual processing in an RSVP task: an attentional blink? J Exp Psychol Hum Percept Perform 18:849-860

Robinson TE, Berridge KC (1993) The neural basis of drug craving: an incentive-sensitization theory of addiction. Brain Res Brain Res Rev 18:247-291

Sacchetti G, Bernini M, Bianchetti A, Parini S, Invernizzi RW, Samanin R (1999) Studies on the acute and chronic effects of reboxetine on extracellular noradrenaline and other monoamines in the rat brain. Br J Pharmacol 128:1332-1338

Strange BA, Dolan RJ (2007) Beta-adrenergic modulation of oddball responses in humans. Behav Brain Funct 3:29

Strange BA, Hurlemann R, Dolan RJ (2003) An emotion-induced retrograde amnesia in humans is amygdala- and beta-adrenergicdependent. Proc Natl Acad Sci U S A 100:13626-13631

Usher M, Cohen JD, Servan-Schreiber D, Rajkowski J, Aston-Jones G (1999) The role of locus coeruleus in the regulation of cognitive performance. Science 283:549-554

Washburn M, Moises HC (1989) Electrophysiological correlates of presynaptic alpha 2-receptor-mediated inhibition of norepinephrine release at locus coeruleus synapses in dentate gyrus. J Neurosci 9:2131-2140

Weinberger NM (1999) Dynamic regulation of receptive fields and maps in the adult sensory cortex. Annu Rev Neurosci 18:129-158

Williams JT, Henderson G, North RA (1985) Characterization of alpha 2 -adrenoceptors which increase potassium conductance in rat locus coeruleus neurones. Neuroscience 14:95-101

Yu AJ, Dayan P (2005) Uncertainty, neuromodulation, and attention. Neuron 46:681-692 